\title{
An Algorithm of Plus-Closures of Loop-Nonnegative Matrices over Idempotent Semirings and its Applications
}

\author{
Wang Zhixi ${ }^{1}$,Wang Yana ${ }^{1}$, Hu Binliang ${ }^{2}$, Liu $\mathrm{Yu}^{3}$ \\ ${ }^{1}$ School of Computer Science and Engineering,Hunan University of Science and Technology,Xiangtan 411201, Hunan, China \\ ${ }^{1}$ Email: zhixiwang@163.com \\ ${ }^{2}$ Colleges of Electromechanical Engineering, Hunan University of Science and Technology, Xiangtan 411201, Hunan, China \\ ${ }^{2}$ Email:blhu@hnust.edu.cn \\ ${ }^{3}$ School of Vocational and Technical,Nanning College of Vocational and Technical, Nanning 530000, Guangxi, China \\ ${ }^{3}$ E-mail: ly102091002@hotmail.com
}

\begin{abstract}
To judge the loop-nonnegativity of a matrix $A$ over an idempotent semiring and compute the plus-closure of $A$ when it is loop-nonnegative, a Plus_Closure_of_Matrix algorithm of complexity $O\left(n^{3}\right)$ is constructed and proved. As a generalization of Floyd algorithm, Warshall algorithm as well as Gauß-Jordan Elimination algorithm on idempotent semirings, this algorithm can also be used to solve some Algebraic Path Problems, Shortest Path Problems and the transitive closures of matrices over idempotent semirings even if the idempotent semirings have no completeness and closeness.
\end{abstract}

Keywords-Idempotent semiring, Loop-nonnegative matrix, Plus-closure, Plus_Closure_of_Matrix Algorithm

\section{INTRODUCTION}

The shortest path problem, calculating the transitive closures of binary relations and finding inverses of Matrices are important issues with a broad background. Floyd algorithms, Warshall algorithms and the Gaussian elimination method are classical algorithms for these three problems respectively [1-3]. Based on the semirings-algebraic theory, these problems were later consolidated as "algebraic path problem". Algebraic path problem and its algorithms have a wide application in the field of network engineering, image processing and pattern recognition.

In 1971, Carré first discovered the similarities of these three problems and proposed their general form Gauß-Jordan elimination for solving them based on semirings algebra theory. The algorithm for solving algebraic path problem boils down to find the Kleene closure of adjacency matrix, i.e., the summation of all non-negative integer powers of the adjacency matrix[4]. Since then, with an in-depth study, Gau $\beta$-Jordan elimination method has been improved from every angle and its application has a gradual expansion from some special semirings first to general cases, and the computing power with the introduction of advanced technologies such as parallel computing has been greatly improved [5-12]. However, the semirings applied to Gau $\beta$ - Jordan elimination method always required completeness and closeness.

This article first proposed the concepts of the Loop-Nonnegative Matrices over Idempotent Semi-rings, then constructed of an algorithm of finding the Plus_Closures_of matrix, i.e., the summation of all positive powers of a matrix. Actually, the algorithm extends the applicable range of Gauß-Jordan elimination method to certain semirings which do not require completeness and closeness.

\section{II . THE ANALYSIS OF \\ PLUS_CLOSURE_OF_MATRIX ALGORITHM AND ITS COMPLEXITY}

Let $R$ be a set containing at least two elements $\overline{0}, \overline{1}$, define the addition + and the multiplication $\times$ on $R$ such that $(R,+, \overline{0})$ is a commutative monoid, and $(R, \times, \overline{1})$ is a monoid with $\overline{0}$ as the zero element, moreover, multiplication $\times$ satisfy the distributive law to addition + . The algebraic system $R=(R,+, \times, \overline{0}, \overline{1})$ defined in this way is called a semiring. Usually, the product $a \times b$ of elements $a, b$ over semiring $R$ is denoted by $a b$. Moreover, the sum of empty sets is denoted by $\overline{0}$, i.e., $\sum \varnothing=\overline{0}$. The summation $a^{+}=\sum_{m=1}^{+\infty} a^{m}$ of $a$ 's all positive integer powers over $R$ is called the plus_closure of $a$.

Similar to the numerical matrices, we can define matrices and their addition and multiplication over semiring $R$. Denote a matrix that the element $(i, j)-$ is $a_{i j}$ over $R$ by $\left(a_{i j}\right)$. Then the set of all $n \times n$ matrices $M_{n}(R)$ on $R$ with regard to matrix addition and multiplication also forms a semiring. This semiring is called an $n \times n$ matrix semiring over $R$. The basic properties of matrices over semirings and the details of semirings can be found in literature [13]. If $A \in M_{n}(R)$, denote the element $(i, j)$ - of $A$ by $A_{i j}$, and let

$$
L(A)=\left\{a_{i_{1} i_{2}} a_{i_{2} i_{3}} \ldots a_{i_{m-1} i_{1}} \mid 1<m, 1 \leq i_{1}, i_{2}, \ldots, i_{m-1} \leq n\right\} .
$$

We define matrices $A^{[k]} \in M_{n}(R)$ recursively as follows:

$$
A_{i j}^{[k]}= \begin{cases}A_{i j} & k=0, \\ A_{i j}^{[k-1]}+A_{i k}^{[k-1]} A_{k j}^{[k-1]} & 1 \leq k \leq n .\end{cases}
$$

Where $k \leq n, k$ is a non-negative integer.

Define the relation $\leq$ over semiring as $a \leq b \Leftrightarrow a+b=a$.

We call $R$ an idempotent semiring if the relation $\leq$ is reflexive. Moreover, the relation is a partial order relation over $R$. Note that the relation $\leq$ over $M_{n}(R)$ can be 
also expressed as $A \leq B \Leftrightarrow A_{i j} \leq B_{i j}$ for $1 \leq i, j \leq n$.

We can conclude that $R$ is idempotent is equivalent to $M_{n}(R)$ is idempotent. The idempotent semirings have a significant application in solving optimazation problems and the classification of regular languages, c.f. [14, 15].

Let $R$ be a semiring, the element $x$ which satisfies $\overline{1} \leq x$ in $R$ is called the non-negative element of $R$. Obviously, $\overline{0}, \overline{1}$ both are the non-negative elements of $R$. For the $n \times n$ matrices $A=\left(a_{i j}\right)$ over $R$, we call that $A$ is loop non-negative if all of the elements of $L(A)$ are non-negative. The following theorem is the main conclusion of this article, and we will consider the proof of this matter in next section.

Theorem 1. Let $A$ be an $n \times n$ matrices over an idempotent semiring $R$, then $A$ is loop non-negative if and only if $A^{[n]} \leq A^{[n]} A^{[n]}$ and $\overline{1} \leq A_{i i}^{[n]}$ for $1 \leq i \leq n$. Furthermore, if $A$ is loop non-negative, then $A^{+}=A^{[n]}$, and for any $1 \leq i, j, k \leq n, A_{i k}^{[k]}=A_{i k}^{[k-1]}, A_{k j}^{[k]}=A_{k j}^{[k-1]}$.

According to theorem 1, we can use the pseudo codes to describe an algorithm as follows about deciding whether the $n \times n$ matrix $A$ is loop non-negative over $R$ and computing the plus-closure $A^{+}$of $A$ when it is loop-nonnegative.

\section{Plus_Closure_of_Matrix algorithm}

Boolean Plus Closure of Matrix (Matrix A, Matrix B)

$\mathrm{n}=$ A.rows " (to obtain the order of matrix A)

$\mathrm{B}=\mathrm{A} "$

" (The first part is to calculate the matrix $A[n]$ )

For $\mathrm{k}=1$ To $\mathrm{n}$

For $\mathrm{i}=1$ (To $\mathrm{n}$ " to calculate the matrix $\mathrm{A}[\mathrm{k}]$ )

$$
\text { For } \mathrm{j}=1 \text { To } n
$$

$$
\text { Next } j
$$$$
B(i, j)=B(i, j)+B(i, k) * B(k, j))
$$

Next i

Next k

"(The second part is to judge the matrix A is loop non-negative or not)

For $\mathrm{i}=1$ To $\mathrm{n}$ Next

If Not Identity $<=\mathrm{B}(\mathrm{i}, \mathrm{i})$ Then Return false "

If Not $\mathrm{B}<=\mathrm{B} * \mathrm{~B}$ Then Return false "

Return true "

End Function

The analysis of the complexity of Plus Closure_of_Matrix algorithm

Plus_Closure_of_Matrix consists of two parts. The first is to compute the matrix $A^{[n]}$, and the second is to decide whether $A$ is loop-nonnegative with respect of $A^{[n]}$. Taken the assignment, addition, multiplication and comparison operations of the elements of the semirings as a basic operation respectively, we can conclude that the first part needs $3 n^{3}+n^{2}$ times of basic operations (including an assignment of matrices, $n^{3}$ times addition, multiplication and assignment of elements of the semirings), The second part takes up at most $2 n^{3}+n^{2}+n$ times of the basic operations (including a multiplication of matrices, a comparison of matrices and at most $n$ times of comparisons of elements of semirings). Hence, the time complexity of the algorithm is $O\left(n^{3}\right)$.

\section{PROOF OF THE PLUS CLOSURE OF MATRIX ALGORITHM}

In this section, we assume that $R$ is always an idempotent semiring, $n$ is a given positive integer and $A=\left(a_{i j}\right) \in M_{n}(R)$. For any positive integer $k$ and non-negative integer $t$, denote that the length of all the sequences, which are consisted of positive integers that is no more than $k$ is $t$ by $I_{t}^{k}$. i.e.,

$$
I_{t}^{k}=\left\{\begin{array}{lr}
\{\varepsilon\}, & t=0, \\
\left\{i_{1} i_{2} \ldots i_{t} \mid 1 \leq i_{1}, \ldots, i_{t} \leq k\right\}, & t>0 .
\end{array}\right.
$$

Where $\varepsilon$ is the empty sequence. The length of sequence $\alpha$ is denoted by $|\alpha|$, and the number of $k$ 's included in $\alpha$ by $|\alpha|_{k}$. For any $\alpha=i_{1} i_{2} \ldots i_{t} \in I_{t}^{n}$ with $t \geq 2$, let $A_{\alpha}=a_{i_{1} i_{2}} a_{i_{2} i_{3}} \ldots a_{i_{t-1} i_{t}}$, then $L(A)=\left\{A_{i \alpha i} \mid 1 \leq i \leq n, \alpha \in I_{t}^{n}, 0 \leq t\right\}$. For any positive integer $m$, the elements $(i, j)-$ of matrices $A^{m}$ and $A^{(m)}=\sum_{i=1}^{m} A^{i} \quad$ can be expressed as:

$$
A_{i j}^{m}=\sum_{\alpha \in I_{m-1}^{n}} A_{i \alpha j}, \quad A_{i j}^{(m)}=\sum_{t=1}^{m}\left(\sum_{\alpha \in I_{t-1}^{n}} A_{i \alpha j}\right) .
$$

We need Lemma 2 to Lemma 8 as follows to prove the Plus_Closure_of_Matrix algorithm, i.e., the Theorem 1.

Lemma 2. Let $a, b, c, d, x_{i} \in R$ with $1 \leq i \leq m$. then the following conditions hold:

( i ) $a \leq \sum_{i=1}^{m} x_{i}$ if and only if $a \leq x_{i} \quad(i=1,2, \ldots, m)$.

(ii) If there exists an integer $i$ such that $x_{i} \leq a$, then $\sum_{i=1}^{m} x_{i} \leq a$.

(iii) If $a \leq b, c \leq d$, then $a c \leq b d$.

(iv) If $b$ is non-negative. Then $a c \leq a b c$.

Proof. ( i ) Obviously, $a \leq \sum_{i=1}^{m} x_{i}$ is included in $a \leq x_{i}$ with $1 \leq i \leq m$. Conversely, assume that $a \leq \sum_{i=1}^{m} x_{i}$. From the fact that the commutativity and idempotency of addition over $R$, we can obtain that for each $x_{i}$,

$$
a=a+\sum_{i=1}^{m} x_{i}=a+\sum_{i=1}^{m} x_{i}+x_{i}=a+x_{i} .
$$


(ii) Assume that there exists an integer $i$ such that $x_{i} \leq a$. We may assume that $i=1$. Then

$$
a+\sum_{i=1}^{m} x_{i}=\left(a+x_{1}\right)+\sum_{i=2}^{m} x_{i}=\sum_{i=1}^{m} x_{i} .
$$

(iii) Assume $a \leq b, c \leq d$. By applying the idempotency of $R$, then we have:

$$
\begin{aligned}
a c+b d & =(a+b)(c+d)+b d=a c+a d+b c+b d+b d \\
& =a c+a d+b c+b d=(a+b)(c+d)=a c .
\end{aligned}
$$

(iv) Let $b$ is non-negative, then by (iii), we can conclude that $a=a \overline{1} \leq a b$, therefore, $a c \leq a b c$.

Corollary 3. Assume that $i, j$ are positive integers with $i, j, k \leq n, \quad \alpha \in I_{t}^{k}$ with $t \geq 0$. If for any $1 \leq i \leq n, A_{i i}^{(k)}$ is non-negative, then there exists a subsequence $\beta$ of $\alpha$ with $|\beta| \leq k$ such that $A_{i \beta j} \leq A_{i \alpha j}$. In particular, if $\min (i, j) \leq k$, then the length of $\beta$ of $\alpha$ as above can be choosed as $|\beta| \leq k-1$.

Proof. Assume that $A_{i i}^{(k)}$ is non-negative for any $1 \leq i \leq n$. Let $\alpha=i_{1} i_{2} \ldots i_{t}$. If $t \leq k, \beta=\alpha$ satisfies the condition. If $t>k$, necessarily there exist $1 \leq p<q \leq t$ and $q-p \leq k$ such that $i_{p}=i_{q}$, therefore, $\overline{1} \leq A_{i_{p} i_{p+1} \ldots i_{q-1} i_{p}}=A_{i_{p} i_{p+1} \ldots i_{q-1} i_{q}}$. Let $\beta=i_{1} i_{2} \ldots i_{p} i_{q+1} \ldots i_{t}$, then $|\beta|<|\alpha|$, by (iv) of Lemma 2 , we can conclude that:

$$
\begin{aligned}
A_{i \beta j} & =A_{i i_{1} i_{2} \ldots i_{p}} A_{i_{q} i_{q+1} \ldots i_{t} j} \\
& \leq A_{i i_{1} i_{2} \ldots i_{p}} A_{i_{p} i_{p+1} \ldots i_{q-1} i_{q}} A_{i_{q} i_{q+1} \ldots i_{t} j} \\
& =A_{i \alpha j} .
\end{aligned}
$$

If $|\beta| \leq k$, then the proof is completed. Otherwise, take $\beta$ as a new $\alpha$ and then repeat the procedures above. Clearly, such a $\beta$ satisfying the conditions can be found.

In particular, when $\min (i, j) \leq k$ take $\beta$ obtained from the procedures above as a new $\alpha$, then $t \leq k$. And if $t \leq k-1$, we can choose $\beta=\alpha$. If $t=k$, assume $i_{0}=i$, $i_{k+1}=j$, then necessarily there exists $0 \leq p<q \leq k+1$ such that $i_{p}=i_{q}$, therefore, $\overline{1} \leq A_{i_{p} i_{p+1} \ldots i_{q-1} i_{p}}=A_{i_{p} i_{p+1} \ldots i_{q-1} i_{q}}$. Now, choose $\beta=i_{1} i_{2} \ldots i_{p} i_{q+1} \ldots i_{k}$, then we can also obtain $|\beta| \leq k-1$.

Lemma 4. $A$ is loop-nonnegative if and only if $A_{i i}^{(n)}$ is non-negative for any $1 \leq i \leq n$.

Proof. For any $1 \leq i \leq n$, from the expression of $A_{i i}^{(n)}$, we conclude that $A_{i i}^{(n)}$ is the sum of finite elements in set $L(A)$. If $A$ is loop-nonnegative, then by ( $\mathrm{i}$ ) of Lemma 2, $A_{i i}^{(n)}$ is non-negative. Conversely, assume that for any $1 \leq i \leq n, A_{i i}^{(n)}$ is non-negative. Recall that for any elements $A_{i \alpha i}$ of $L(A)$, with $\alpha \in I_{t}^{n}, t \geq 0,1 \leq i \leq n$. By Corollary 3, we can conclude that there exists a subsequence $\beta$ of $\alpha$ with $|\beta| \leq n-1$ such that $A_{i \beta i} \leq A_{i \alpha i}$. Note that $A_{i \beta i}$ is one of the expression of $A_{i i}^{(n)}$, by ( $\mathrm{i}$ ) of Lemma 2 , we see that $A_{i \beta i}$ is non-negative, thus $A_{i \alpha i}$ is non-negative.

Lemma 5. If $A^{[n]} \leq A^{[n]} A^{[n]}$ and for any $1 \leq i \leq n, \overline{1} \leq A_{i i}^{[n]}$, then $A$ is loop non-negative.

Proof. Assume that $A^{[n]} \leq A^{[n]} A^{[n]}$ and for any $1 \leq i \leq n$, $\overline{1} \leq A_{i i}^{[n]}$. By the definition of $A^{[n]}$ and (ii) of Lemma 2, we can obtain that $A^{[n]} \leq A^{[n-1]} \leq \cdots \leq A^{[0]}=A=A^{(1)}$.

Assume that the relation $A^{[n]} \leq A^{(m)}$ holds when $m \geq 1$. Since $A^{[n]} \leq A^{[n]} A^{[n]}$, we can conclude that $A^{[n]}=A^{[n]}+A^{[n]} A^{[n]} \leq A+A A^{(m)}=A^{(m+1)}$ by ( i ) (iii) of Lemma 2 and the idempotency. This means that for any $m \geq 1$, $A^{[n]} \leq A^{(m)}$. It follows that $\overline{1} \leq A_{i i}^{[n]} \leq A_{i i}^{(n)}$ with $1 \leq i \leq n$. From Lemma 4 we can conclude that $A$ is loop non-negative.

Lemma 6. If $A$ is loop non-negative, then $A^{+}=A^{(n)}$. Proof. For any positive integer $k, i, j$ with $i, j \leq n$ consider the typical term $A_{i \alpha j}$ with $\alpha \in I_{t-1}^{n}, 1 \leq t \leq n+k$ in the expression of $A_{i j}^{(n+k)}$. By Corollary 3 and Lemma 4, we obtain that there exists a subsequence $\beta$ with $|\beta| \leq n-1$ such that $A_{i \beta j} \leq A_{i \alpha j}$. Since $A_{i \beta j}$ is an item of $A_{i j}^{(|\beta|+1)}$, so it is also an item of $A_{i j}^{(n)}$. Hence, $A_{i j}^{(n)} \leq A_{i \alpha j}$. Thus, by ( $\mathrm{i}$ ) of Lemma 2, then we have $A_{i j}^{(n)} \leq A_{i j}^{(n+k)}$, therefore, $A^{(n)} \leq A^{(n+k)}$. From (ii) of Lemma 2 we can also conclude $A^{(n+k)} \leq A^{(n)}$. This implies that for any positive integer $k$, we have $A^{(n)}=A^{(n+k)}$, hence, $A^{+}=A^{(n)}$.

For any non-negative integer $t$ and positive integer $k$ with $k \leq n$, we denote

$$
S_{t}^{k}=\left\{\alpha \in I_{t}^{k}|\alpha|_{k} \geq 1\right\}, \quad T_{t}^{k}=\left\{\left.\alpha \in I_{t}^{k}|| \alpha\right|_{k}=1\right\} .
$$

Clearly, $S_{0}^{k}=T_{0}^{k}=\varnothing$.

Lemma 7. If $A$ is loop non-negative, then for any positive integers $i, j, k$ with $i, j, k \leq n$,

$$
\sum_{t=0}^{k} \sum_{\alpha \in S_{t}^{k}} A_{i \alpha j}=\sum_{t=1}^{k} \sum_{\alpha \in T_{t}^{k}} A_{i \alpha j} .
$$

Proof. For any $\alpha=i_{1} \ldots i_{t} \in S_{t}^{k} \backslash T_{t}^{k}$, let

$$
p=\min \left\{r \mid i_{r}=k\right\}, q=\max \left\{r \mid i_{r}=k\right\}, \quad \beta=i_{1} \ldots i_{p} i_{q+1} \ldots i_{t-1} .
$$

Then we have $\beta \in T_{t+p-q}^{k}$. Since $A$ is loop non-negative, and by (iv) of Lemma 2. we can conclude that $A_{i \beta j} \leq A_{i \alpha j}$. Moreover, by ( $\mathrm{i}$ ) of Lemma 2 and the definition of relation $\leq$ as well as the fact that the sum of empty set is $\overline{0}$, it follows that:

$$
\sum_{t=0}^{k} \sum_{\alpha \in S_{t}^{k}} A_{i \alpha j}=\sum_{t=1}^{k} \sum_{\alpha \in T_{t}^{k}} A_{i \alpha j}+\sum_{t=1}^{k} \sum_{\alpha \in S_{t}^{k} \backslash T_{t}^{k}} A_{i \alpha j}=\sum_{t=1}^{k} \sum_{\alpha \in T_{t}^{k}} A_{i \alpha j} .
$$


Lemma 8. If $A$ is loop non-negative, then for any $1 \leq i, j \leq n, A_{i j}^{[k]}=P A_{i j}^{[k]}$, where $P A_{i j}^{[k]}=\sum_{t=0}^{k} \sum_{\alpha \in I_{t}^{k}} A_{i \alpha j}$.

Proof. The proof is by mathematical induction. Obviously, $A_{i j}^{[0]}=P A_{i j}^{[0]}$. Assume that $A_{i j}^{[k-1]}=P A_{i j}^{[k-1]}$ for $k \geq 1$. We have to show that $A_{i j}^{[k]}=P A_{i j}^{[k]}$. By the definition of $A_{i j}^{[k]}$ and induction hypothesis, we can conclude that

$$
\begin{aligned}
A_{i j}^{[k]} & =P A_{i j}^{[k-1]}+P A_{i k}^{[k-1]} \times P A_{k j}^{[k-1]} \\
& =\sum_{t=0}^{k-1} \sum_{\alpha \in I_{t}^{k-1}} A_{i \alpha j}+\sum_{m=0}^{k-1} \sum_{\alpha \in I_{m}^{k-1}} A_{i \alpha k} \times \sum_{t=0}^{k-1} \sum_{\beta \in I_{t}^{k-1}} A_{k \beta j} \\
& =\sum_{t=0}^{k-1} \sum_{\alpha \in I_{t}^{k-1}} A_{i \alpha j}+\sum_{m, t=0}^{k-1} \sum_{\alpha \in I_{m}^{k-1}, \beta \in I_{t}^{k-1}} A_{i \alpha k} A_{k \beta j} \\
& =\sum_{t=0}^{k-1} \sum_{\alpha \in I_{t}^{k-1}} A_{i \alpha j}+\sum_{m, t=0}^{k-1} \sum_{\alpha \in I_{m}^{k-1}, \beta \in I_{t}^{k-1}} A_{i \alpha k \beta j} \\
& =\sum_{t=0}^{k-1} \sum_{\alpha \in I_{t}^{k-1}} A_{i \alpha j}+\sum_{t=1}^{k} \sum_{\alpha \in T_{t}^{k}} A_{i \alpha j}+\sum_{t=1}^{k-1} \sum_{\alpha \in T_{k}^{k} I_{t}^{k-1}} A_{i \alpha j}
\end{aligned}
$$

For any $\gamma \in I_{t}^{k-1}$ with $t \geq 0$, by Corollary 3 and Lemma 4, we can obtain that there exists a subsequence $\beta$ of $\gamma$ with $|\beta| \leq k-1$ such that $A_{i \beta j} \leq A_{i \gamma j}$. Since

$$
\beta \in \bigcup_{t=0}^{k} I_{t}^{k-1}
$$

and by ( ii ) of Lemma 2 ,

$$
\sum_{t=0}^{k-1} \sum_{\alpha \in I_{t}^{k-1}} A_{i \alpha j} \leq \sum_{t=0}^{k} \sum_{\alpha \in I_{t}^{k-1}} A_{i \alpha j} .
$$

Furthermore, from the argument of ( i ) of Lemma 2, we have

$$
A_{i j}^{[k]}=\sum_{t=0}^{k} \sum_{\alpha \in I_{t}^{k-1}} A_{i \alpha j}+\sum_{t=0}^{k} \sum_{\alpha \in T_{t}^{k}} A_{i \alpha j}+\sum_{t=1}^{k-1} \sum_{\alpha \in T_{k}^{k} I_{t}^{k-1}} A_{i \alpha j} .
$$

For any $\gamma \in T_{k}^{k} I_{t}^{k-1}$ with $1 \leq t \leq k-1$, since $T_{k}^{k} I_{t}^{k-1} \subset I_{k+t}^{k}$, from the fact of Corollary 3 and Lemma 4, clearly, there exists a subsequence $\beta$ with $|\beta| \leq k$ of $\gamma$ such that $A_{i \beta j} \leq A_{i \gamma j}$. Since

$$
\beta \in\left(\bigcup_{t=0}^{k} I_{t}^{k-1}\right) \cup\left(\bigcup_{t=1}^{k} T_{t}^{k}\right)
$$

and by ( ii ) of Lemma 2, we have

$$
\sum_{t=0}^{k} \sum_{\alpha \in I_{t}^{k-1}} A_{i \alpha j}+\sum_{t=1}^{k} \sum_{\alpha \in T_{t}^{k}} A_{i \alpha j} \leq A_{i \gamma j} .
$$

And by ( $\mathrm{i}$ ) of Lemma 2, meaning that

$$
\sum_{t=0}^{k} \sum_{\alpha \in I_{t}^{k-1}} A_{i \alpha j}+\sum_{t=1}^{k} \sum_{\alpha \in T_{t}^{k}} A_{i \alpha j} \leq \sum_{t=1}^{k-1} \sum_{\alpha \in T_{k}^{k} I_{t}^{k-1}} A_{i \alpha j} .
$$

Further by the definition of relation $\leq$, we can conclude that

$$
A_{i j}^{[k]}=\sum_{t=0}^{k} \sum_{\alpha \in I_{t}^{k-1}} A_{i \alpha j}+\sum_{t=1}^{k} \sum_{\alpha \in T_{t}^{k}} A_{i \alpha j} .
$$

Then from the fact of Lemma 7, we can also get

$$
A_{i j}^{[k]}=\sum_{t=0}^{k} \sum_{\alpha \in I_{t}^{k-1}} A_{i \alpha j}+\sum_{t=0}^{k} \sum_{\alpha \in S_{t}^{k}} A_{i \alpha j} .
$$

Since
Therefore,

$$
I_{t}^{k}=I_{t}^{k-1} \cup S_{t}^{k} \text { for } \quad t \geq 0
$$

$$
A_{i j}^{[k]}=\sum_{t=0}^{k}\left(\sum_{\alpha \in I_{t}^{k}} A_{i \alpha j}\right)=P A_{i j}^{[k]} .
$$

Proof of Theorem 1. Assume that $A$ is loop-nonnegative, by Lemma 5 we can conclude that to prove Theorem 1, we just need to prove $A^{+}=A^{[n]}, A^{[n]} \leq A^{[n]} A^{[n]}$, and for any $1 \leq i \leq n, \overline{1} \leq A_{i i}^{[n]}$. Noting that $P A_{i j}^{[n]}=A_{i j}^{(n+1)}$, then from the argument of Lemma 6 and Lemma 8 as well as the indempotency, we can obtain that

therefore,

$$
A^{+}=A^{(n)}=A^{(n+1)}=P A^{[n]}=A^{[n]},
$$

$$
\begin{aligned}
A^{[n]} & =A^{(n)}=A^{(2 n)}=A+A^{(n)} A^{(n)} \\
& \leq A^{(n)} A^{(n)}=A^{[n]} A^{[n]} .
\end{aligned}
$$

By Lemma 4 and $A^{(n)}=A^{[n]}$, we can conclude that for any $1 \leq i \leq n, \quad \overline{1} \leq A_{i i}^{(n)}=A_{i i}^{[n]}$.

Then the proof is completed.

\section{THE APPLICATION OF THE PLUS_CLOSURE_OF_MATRIX ALGORITHM}

Let $R$ be a semiring, $G=(V, E)$ be a (directed) graph, where $V=\{1,2, \cdots, n\}$ is the vertices set, and $E \subseteq V \times V$ is the edges set. The set of all paths from vertex $i$ to vertex $j$ is denoted by $i \rightarrow j$. The length of the path $p$ in $G$ is denoted by $|p|$. For a given function $w: E \rightarrow R$, we can define the weighted graph $G=(V, E, w)$ over $R$. The image $w(e)$ of function $w$ is called the value of $e$. The adjacency matrix $A(G)=\left(w_{i j}\right)$ of $G$ is defined as follows:

$$
w_{i j}= \begin{cases}w((i, j)), & (i, j) \in E, \\ 0, & (i, j) \notin E .\end{cases}
$$

If $p=\left(p_{0}, p_{1}, \ldots, p_{k}\right) \in i \rightarrow j$, then we say that $w(p)=\prod_{i=0}^{k} w\left(p_{i}\right)$ is the value of $p$. And the distance from vertex $i$ to vertex $j$ is defined as $d_{i j}=\sum_{p \in i \rightarrow j} w(p)$.

\section{The Algebraic path problems}

Algebraic path problems over a semiring is actually to find the distance between any two vertices $i$ to $j$ over the weighted graph $G=(V, E, w)$ of $R$. If there exist solutions to the algebraic path problems, we call that the matrix $S(G)=\left(d_{i j}\right)$ is the solution matrix of $G$. Obviously, if $(A(G))^{+}$exists, then $S(G)=(A(G))^{+}$. Consequently, when $R$ is an idempotent semiring and $A(G)$ is a loop non-negative matrix, Plus Closure of Matrix algorithm is an effective method to find the solution matrix $S(G)$ of $G$. Actually, if the process of computing $A^{*}=I_{n}+A^{+}$can be added to the algorithm, where $I_{n}$ is the $n \times n$ identity 
matrix, then we can obtain the Gauß-Jordan elimination method. In this case, there is no need to require completeness and closeness of $R$.

\section{The algebraic path problem over inclines}

We say that semi-ring $R$ is an incline if for any $x \in R$, $\overline{1}+x=\overline{1}$. Incline is an idempotent semiring that all elements are non-negative[16]. Actually, Plus_Closure_of_Matrix algorithm over inclines is the Gau $\beta$-Jordan elimination method that Mohri proposed in literature [11].

\section{The shortest path problem of over totally ordered idempotent semirings}

If $R$ is an idempotent semiring and the partial order relation $\leq$ over $R$ is a total order relation, we say that $R$ is a totally ordered idempotent semiring. The set $\Delta$ which consists of non-negative real numbers and $+\infty$ with regard to the minimizing operation and the addition of numbers form a totally ordered incline.

For any two vertices $i, j$ of $G$, note that even if $d_{i j}$ (the distance between $i$ and $j$ )may exists, the shortest path from $i$ to $j$ may still not. When $R$ is a totally ordered idempotent semiring and $A(G)$ is a loop non-negative matrix, then by the totally order nature of the partial order relation $\leq$ over $R$, necessarily there exists a shortest path from $i$ to $j$. Therefore, Modeled on Floyd algorithms, we can constructe an algorithm based on Plus_Closure_of_Matrix algorithm to solve the shortest path problems over $\bar{R}$, so that the algorithm in the case of $R=\Delta$ is the Floyd algorithm.

\section{Matrix of transitive closure}

Assume that $A=\left(a_{i j}\right) \in M_{n}(R)$. Let

$$
\Omega(A)=\left\{A^{\prime} \in M_{n}(R) \mid A^{\prime} \leq A, A^{\prime} \leq A^{\prime} A^{\prime}\right\} .
$$

$A^{t}$ is called the transitive closure of $A$ if for any $A^{\prime} \in \Omega(A)$, $A^{\prime} \leq A^{t}$ holds. It is easy to prove that when $R$ is an idempotent semiring and $A$ is a loop non-negative matrix, $A^{+}$is the transitive closure of $A$, i.e., $A^{t}=A^{+} . A^{t}=A^{+}$. Therefore, the Plus_Closure_of_Matrix algorithm can be used to calculate the transitive closure of the loop-nonnegative matrices over idempotent semirings.

Obviously, Binary Boolean semiring $B=(\{0,1\}, \vee, \wedge, 0,1)$ is also an incline. Since the adjacent matrix $A(G)$ of a binary relation $G$ of finite sets $V$ is a matrix over $B$, and its transitive closure is the adjacent matrix of $G$ 's transitive closure. Therefore, the Plus Closure of Matrix algorithm can be used to calculate the transitive closure of a binary relation of finite sets. Actually, Plus Closure of Matrix algorithm over $B$ is the Warshall algorithm.

\section{ACKNOWLEDGMENT}

This work is supported by National Nature Science Foundation of China (No:61272196), Hunan Provincial Natural Science Foundation of China ( No: 10JJ3067), and Scientific Research Fund of Hunan Provincial Education Department (No: 11C0534).

\section{REFERENCE}

[1] Floyd R W. Algorithm 97: Shortest path[J]. Communications of the ACM, 1962, 5(6): 345

[2] Warshall S. A Theorem on Boolean Matrices[J]. Journal of the ACM, 1962, 9(1): 11-12.

[3] Dasgupta S. Papadimitriou C. Vazirani U. Algorithms[M], The McGraw-Hill Companies, Inc., New York, 2008.

[4] Carré B A. An algebra for network routine problems[J]. Journal of the Institute of Mathematical and Its Applications, 1971, 7: 273-294.

[5] Zimmermann U. Linear and combinatorial optimization in ordered algebraic structures[J]. Annals of Discrete Mathematics, 1981, 10: $1-380$

[6] Gunter-Roze G. A systolic array algorithm for the algebraic path problem (Shortest path; Matrix inversion) [J]. Computing, 1985, 34: 191-219.

[7] Tsai H, Horng S, Tsai S, et al. Solving an algebraic path problem and some related graph problems on a hyper-bus broadcast network[J]. IEEE Transactions on Parallel and Distributed Systems, 1997, 8 (12): 1226-1235.

[8] Djamegni C T, Quinton P, Rajopadhye S, et al. Derivation of systolic algorithms for the algebraic path problem by recurrence transformations[J]. Parallel Computing, 2000, 26 (17): 1429-1445.

[9] Takahashi A, Sedukhin S. Parallel Blocked Algorithm for solving the algebraic path problem on a matrix processor[J]. Lecture Notes in Computer Science, 2005, 3726: 786-795.

[10] Lluch-Lafuente A, Montanari A. Quantitative $\mu$-calculus and CTL defined over constraint semirings[J]. Theoretical Computer Science, 2005, 346 (1): 135-160

[11] Mohri M. Semiring frameworks and algorithms for shortest-distance problems[J]. Journal of Automata, Languages and Combinatorics, 2003, 7 (3): 321-350

[12] Penn G. Efficient transitive closure of sparse matrices over closed semirings[J]. Theoretical Computer Science, 2006, 354 (1): 72 - 81 .

[13] Golan J S. Semirings and Their Applications[M]. Kluwer Academic Publishers, Dordrecht, 1999.

[14] Kolokoltsov V N, Maslov V P. Idempotent analysis and its applications[M]. Dordrecht: Kluwer Academic Publishers, 1997.

[15] Polak L. A classification of rational languages by semilattice-ordered monoids[J]. Archiv der Mathematik, 2004, 3153 : 635-647.

[16] Han S, Li H X. Indices and periods of incline matrices[J]. Linear Algebra and Its Applications, 2004, 387: 143-165. 